\title{
The inverse association between relative abundances of oleic acid and arachidonic acid is related to alpha -linolenic acid
}

\author{
Arne Torbjørn Høstmark ${ }^{1 *}$ and Anna Haug ${ }^{2}$
}

\begin{abstract}
Background: Many health effects of oils rich in oleic acid (OA, 18:1 n9) seem to be opposite those of arachidonic acid (AA, 20:4 n6), i.e. concerning cardiovascular risk. In recent studies in humans and in the rat we observed that percentages of $O A$ and $A A$ were inversely related, raising the question of whether the inverse association is a general one, and how it might be explained. In the present work we examine whether percentages of OA and AA are inversely associated in breast muscle lipids of chickens, and whether alpha-linolenic acid (ALA) may be related to the OA/AA ratio.
\end{abstract}

Methods: The study group consisted of 163 chickens. Breast muscle was collected, and the concentration of fatty acids in muscle lipids was determined using gas chromatography. We studied association between fatty acids using bivariate correlations (Pearson) and linear regression. Synthesis of OA from stearic acid (Stear) was estimated using the OA/Stear ratio, and formation of AA from linoleic acid (LA) was estimated by the AA/LA ratio.

Results: We found a strong inverse relationship ( $r=-0.942, p<0.001 ; n=163)$ between \% OA and \% AA in breast muscle lipids of the chickens. There was an inverse association $(r=-0.887, p<0.001)$ between the OA/Stearic acid ratio, estimating Delta9 desaturase, and the AA/LA ratio, estimating desaturases/elongase activities. Furthermore, there was a strong negative association between \% AA and the OA/Stearic acid ratio $(r=-0.925, p<0.001)$, and \% OA correlated negatively $(r=-0.914, p<0.001)$ with the AA/LA ratio. ALA was positively associated $(r=0.956$, $p<0.001)$ with the OA/AA ratio, and this association prevailed when controlling for the other fatty acids. ALA was positively associated $(r=0.857, p<0.001)$ with the OA/Stear ratio, but was negatively related $(r=-0.827, p<0.001)$ to the AA/LA ratio.

Conclusions: The relative abundances of $\mathrm{OA}$ and $\mathrm{AA}$ that are inversely related in muscle lipids of chickens may be explained by a feedback regulation between the synthesis of OA and AA, and related to ALA, which seems to stimulate formation of $\mathrm{OA}$, and inhibit synthesis of $\mathrm{AA}$, but further studies are required to clarify whether this hypothesis is valid.

\section{Background}

It is widely accepted that oleic acid (OA, 18:1 n9), and oleic acid rich foods such as olive oil may have many beneficial health effects. Among such effects are improved insulin sensitivity, and endothelium-dependent flow-mediated vasodilatation [1], lowering of LDL cholesterol [2,3] and an increase in HDL cholesterol [4]. If lipids in LDL are enriched in oleic acid, the particles will

\footnotetext{
* Correspondence: a.t.hostmark@medisin.uio.no

${ }^{1}$ Section of Preventive Medicine and Epidemiology, University of Oslo, Norway, Institute of Health and Society, Box 1130 Blindern, 0318 Oslo, Norway Full list of author information is available at the end of the article
}

be less liable to be oxidized [5], a property that is of significance for the normal metabolism of LDL [6]. Furthermore, intake of oleic acid seems to be associated with reduced blood pressure [7]. Thus, many of the effects of oleic acid may serve to reduce the risk of cardiovascular diseases. Additionally, the fatty acid may have anti-carcinogenic and anti-inflammatory effects [8-10].

Although beneficial effects of oils rich in oleic acid have been reported, the mechanisms by which such oils might have beneficial health effects are still incompletely understood. Various antioxidants present in e.g. virgin 
olive oil, as well as the high content of oleic acid, could partly explain the health effects.

When considering the reported beneficial health effects of oils rich in oleic acid, we previously suggested $[11,12]$ that many of the positive effects would be anticipated if the fatty acid works to counteract effects of arachidonic acid (AA, 20:4 n6). This fatty acid is formed in the body from linoleic acid (LA, 18:2 n6), a major constituent in many plant oils, and is converted by cyclooxygenase and lipoxygenase into various eicosanoids, i.e. prostacyclines, thromboxanes and leukotrienes [13]. AA derived thromboxane $\mathrm{A}_{2}\left(\mathrm{TXA}_{2}\right)$ and leukotriene $\mathrm{B}_{4}$ have strong proinflammatory and prothrombotic properties $[14,15]$. Furthermore, endocannabinoides, which are derived from arachidonic acid, may have a role in adiposity and inflammation [16].

An interaction between oleic acid and arachidonic acid was suggested several decades ago in the rat [17]. More recently, Cicero et al. [5] showed in human subjects that supplementation with a high dose of olive oil for 3 weeks resulted in an increase in LDL oleic acid and a decrease in linoleic and arachidonic acid. Also in chicken breast muscle a negative $18: 1 \mathrm{n} 9$ vs. 20:4 $\mathrm{n} 6$ association was observed [18].

One mechanism by which OA could counteract those of AA is to reduce the relative abundance of AA in serum and tissues. Conceivably, increased supply of oleic acid might reduce that of AA by pure mass action. Inverse regulation could also be effected through more specific metabolic feedback regulation. For example, a reduced percentage of AA would be expected if OA inhibits Delta-6 desaturase, Elongase-5 (Elovl-5) and/or Delta-5 desaturase, the enzymes governing formation of AA from LA. Conversely, inhibition by AA of Delta-9 desaturase should lower percentage $\mathrm{OA}$, and previous studies suggest that this latter mechanism might take place [19].

It seems that the Delta-9 desaturases are of considerable physiological significance. Thus, regulation of the amount of monounsaturated fatty acids (MUFA) has the potential to affect a variety of key physiological variables, such as insulin sensitivity, metabolic rate, adiposity, atherosclerosis, cancer and obesity $[19,20]$.

In accordance with the above considerations, in a rat study we recently reported that percentages of OA and AA were inversely related [11]. Subsequently, we observed a similar inverse association in the phospholipid faction of human sera [12]. These observations raise the question of whether the OA vs. AA relationship is a general one. In the present work we use data obtained in a previous study [21] to extend our previous work by examining whether an inverse OA vs. AA association might also exist in chickens.

Although a direct feedback regulation between the synthesis of OA and AA seems to be involved to explain the appreciable variation in the OA/AA ratio, the possibility exists that this ratio is also governed by other fatty acids. One particular candidate is ALA, the precursor of the endogenous synthesis of EPA, and known to have many health effects [22-24]. Therefore, as part of the present work we included analyses to examine whether ALA might be related to the OA/AA ratio.

\section{Results}

Is there a direct feedback regulation between the synthesis of $O A$ and $A A$ in lipids of chicken breast muscle?

We found a tight inverse relationship $(r=-0.936, p<0.001$; $\mathrm{n}=163$ ) between percentages of OA and AA in breast muscle lipids of the chickens (Figure 1, panel A). There was also a strong inverse relationship between the product/precursor index estimating synthesis of $\mathrm{OA}$ from stearic acid, and the product/precursor estimate of AA synthesis from LA $(r=-856, p<0.001$, Figure 1 , panel B). In addition, we found strong inverse associations between $\% \mathrm{AA}$ and the OA/Stearic acid ratio, an estimate of OA synthesis $(r=-0.859, p<0.001$, panel $C)$, and also between $\%$ AA and the AA/LA ratio, estimating AA synthesis from LA $(r=-0.884, p<0.001$, panel $\mathrm{D})$. Diet type did not influence the association; when analyzing the relationships shown in Figure 1 in each diet subgroup separately (see Methods), we obtained highly significant correlations $(\mathrm{r}>0.8$; in many groups: $\mathrm{r}>0.9)$; with $\mathrm{p}<0.001$ in all subgroups (results not shown otherwise).

\section{Is the variation in the OA/AA ratio related to ALA?}

The results shown in Figure 1 imply a considerable variation in the OA/AA ratio in breast muscle lipids of the chickens. As shown in Figure 2, top panel, there was a strong positive association between ALA and the OA/ AA ratio $(\mathrm{r}=0.956, \mathrm{p}<0.001, \mathrm{n}=163)$.

Using multiple linear regression we next studied whether the ALA vs. OA/AA ratio association would persist when controlling for other fatty acids. As shown in Table 1, ALA was still significantly $(\mathrm{p}<0.001)$ associated with the OA/AA ratio when controlling for diet type and all of the other fatty acids measured (Model 4, Table 1). As judged from the magnitude of the standardized regression coefficient, the association was weakened in the adjusted model.

\section{Is ALA a stimulator of OA synthesis and/or an inhibitor of} AA synthesis?

A positive relationship between ALA and the OA/AA ratio (Figure 2, upper panel) would be expected if ALA is a stimulator of the synthesis of OA and/or an inhibitor of the synthesis of AA. We therefore studied the association between ALA and the synthesis of OA and AA, as assessed by the product/precursor ratio. Indeed, 

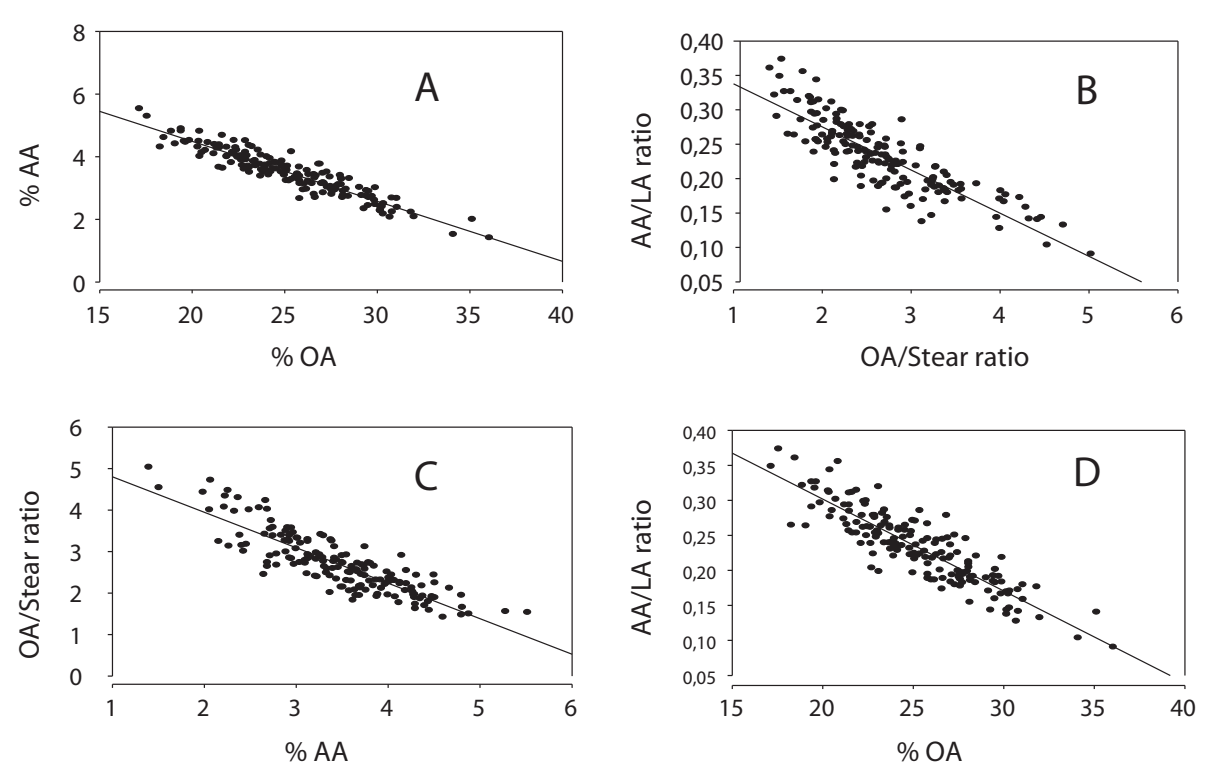

Figure 1 Relationship between estimates of $O A$ and AA formation. Relationship between relative amounts of OA and AA (Panel $\mathbf{A}$ ) in breast muscle lipids of chickens fed various diets containing linseed oil, pooled data analysis (see Methods). Panel B: association between estimates of $\mathrm{OA}$ and AA synthesis, i.e. product/precursor ratios. Panel C: Relationship between \% AA and product/precursor estimate of OA synthesis. Panel $\mathbf{D}$ : relationship between \% OA and product/precursor estimate of AA formation from LA. OA = oleic acid (18:1 n9); Stear = stearic acid (18:0); $A A=$ arachidonic acid (20:4 n6); LA = linoleic acid (18:2 n6). Note broken axes. Coefficients of correlation in panels $\mathbf{A}, \mathbf{B}, \mathbf{C}$, and $\mathbf{D}$ were: $-0.936(p<0.001),-0.856$ $(p<0.001),-0.859, p<0.001)$, and $-0.884, p<0.001)$, respectively. When analyzing the same relationships in each of the diet subgroups (see Methods) separately, we obtained highly significant correlation coefficients $(r>0.8 ; p<0.001)$ in all subgroups.

there was a highly significant positive association $(r=0.857$, $\mathrm{p}<0.001$ ) between ALA and the OA/Stear ratio, estimating OA synthesis (Figure 2, middle panel). Furthermore, we observed a negative association $(r=-0.827, \mathrm{p}<0.001)$ between ALA and the AA/LA ratio, estimating AA synthesis from LA (Figure 2, lower panel). Multiple linear regression analyses with control for diet type and all of the other fatty acids showed that these associations prevailed (ALA vs. OA/Stear: $\mathrm{t}=6.2, \mathrm{p}<0.001$; ALA vs AA/LA: $\mathrm{t}=-2.4, \mathrm{p}=0.021$, results not shown otherwise). When analyzing the associations shown in Figure 2 in each diet subgroup separately (see Methods), we obtained highly significant $(\mathrm{p}<0.001)$ positive and negative, respectively, correlations $(r>0.8$; in many subgroups $r>0.9)$ in all subgroups (results not shown otherwise).

\section{Discussion}

The present work indicates that there is a close inverse relationship between percentages of $\mathrm{OA}$ and $\mathrm{AA}$ acid in breast muscle lipids of chickens. The result supports our previous reports showing a similar inverse relationship between \% OA and \% AA in serum phospholipids of young, healthy subjects [12], and also found in total serum lipids of rats [11] It would appear, therefore, that the observed inverse \% OA vs. \% AA relationship is a general one.

In the rat, an interaction between $\mathrm{OA}$ and linoleic acid, the precursor of AA, was reported several decades ago [17], and in a multi-center randomized cross-over study involving 200 healthy European subjects, Cicero et al. more recently [5] showed that a 3 weeks supplementation with olive oil resulted in an increase in OA in LDL and a decrease in LA and AA. The increase in the $\mathrm{OA} / \mathrm{LA}$ ratio was accompanied by reduced levels of isoprostanes, biomarkers of oxidative stress.

The finding of a negative correlation between $\mathrm{OA}$ and AA also in mice treated with perfluorinated fatty acids [25], zenobiotics used as surfactants in various industrial products, would seem in support of the contention that the inverse relationship between this couple of fatty acids exists in many species.

By pure mass action, increased supply of OA might replace AA in various compartments, such as in the lipids of cell membranes. Conceivably, increase in the percentage of one particular fatty acid must be accompanied by a reduction in the percentage of one or more of the other fatty acids. This type of mechanism was previously suggested by the results of a previous diet trial in chicken [18].

It has been suggested that $\mathrm{OA}$ is a weak competitive inhibitor of cyclooxygenase [26], which catalyzes conversion of the C20 PUFAs AA and EPA into prostaglandins, thromboxanes and leucotrienes [13], and OA might accordingly increase AA and EPA.

Another mechanism serving to explain the inverse relationship could be that $\mathrm{OA}$ acts as an inhibitor of Delta-5/6 desaturases and/or Elongase- 5 so as to reduce 


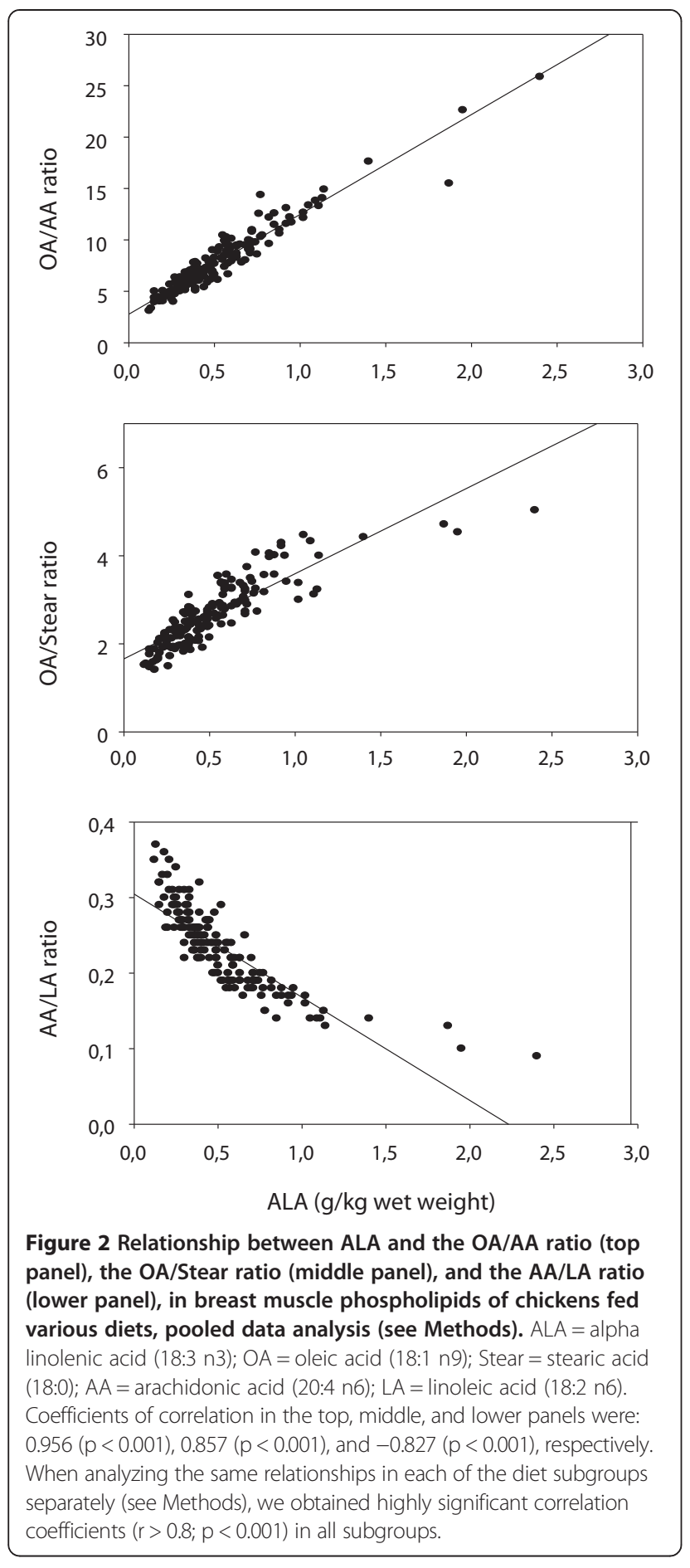

the formation of AA. Additionally, the possibility exists that AA might inhibit the formation of OA by inhibition of Delta-9 desaturase. Possibly, inhibition by AA of Delta-9 desaturase gene transcription might be involved, since previous studies suggest that PUFAs of both the $\mathrm{n} 6$ and $\mathrm{n} 3$ families can inhibit this transcription [20]. The inverse association between \% OA and the AA/LA ratio, used as an estimate of AA synthesis from LA, the
Table 1 Association between ALA (independent variable under investigation) and the $O A / A A$ ratio (dependent variable), multiple linear regression

\begin{tabular}{lllll}
\hline Model & B (SE) & Beta & $\mathbf{t}$ & $\mathbf{p}$ \\
\hline 1 & $9.71(0.23)$ & 0.956 & 41.5 & $<0.001$ \\
2 & $9.49(0.22)$ & 0.934 & 42.3 & $<0.001$ \\
3 & $12.50(0.79)$ & 1.231 & 15.9 & $<0.001$ \\
4 & $8.42(1.43)$ & 0.828 & 5.9 & $<0.001$
\end{tabular}

Dependent variable $=O A / A A$ ratio. $B=$ unstandardized regression coefficient, Beta $=$ standardized coefficient. Model 1 is without adjustment; Model 2: adjusted for 20:3 n3 + 20:5 n3 + 22:5 n3 + 22:6n3; Model 3= Model 2 + 18:2 n6 + 18:3 n6 + 20:2 n6 + 20:3 n6; Model 4= Model 3 + 14:0 + 14:1 c9+15:0+16:0+16:1 c9+ $17: 0+18: 0+18: 2 c 11+18: 2 n 6+20: 0+20: 1 n 9+20: 2 n 6+20: 3 n 6+$ unidentified 18:1 isomers ( minor amounts) + diet type.

inverse association between \% $\mathrm{AA}$ and the OA/Stear ratio, estimating OA synthesis, as well as the inverse association between the ratios estimating synthesis of $\mathrm{OA}$ and $\mathrm{AA}$, respectively, i.e. the $\mathrm{OA} /$ Stear ratio and the AA/LA ratio, appear to be in accordance with this hypothesis.

The linear inverse association between relative abundances of $\mathrm{OA}$ and $\mathrm{AA}$ in chicken muscle lipids indicates that the OA/AA ratio may vary appreciably. Furthermore, the present multiple regression analyses suggest that ALA might at least partly govern the ratio between $\mathrm{OA}$ and $\mathrm{AA}$ in muscle lipids of chickens. The association between ALA and the OA/AA ratio prevailed with high significance when controlling for each of the several fatty acids measured. The association was attenuated when controlling for all of the fatty acids measured (Table 1, regression Model 4), as judged by the magnitude of the standardized regression coefficient. However, in this case we might have an overadjustment bias [27], due to control for some fatty acids which are intermediate variables (or a descending proxy for an intermediate variable) on a causal path from exposure to outcome. One example is LA which is the precursor of AA, a component of the outcome variable. On the other hand, some of the fatty acids entered among the independents may not solely be intermediates, but also serve as regulators of enzymes involved in the metabolism of fatty acids in both the $\mathrm{n} 3$ and $\mathrm{n} 6$ families. Since the regulatory function of fatty acids is not completely clarified, we have included all of the measured fatty acids in our regression analyses. Although the highly significant positive association between ALA and the OA/AA ratio prevailed also when including other fatty acids in the regression model, we cannot rule out the possibility that our results might be explained by as yet unknown covariates, and by the several intercorrelations among the fatty acids.

The observed tight positive coupling between ALA and the product/precursor estimate used for OA synthesis, as well as the strong negative association between ALA and the AA/LA ratio used to estimate AA synthesis from LA, 
seem to be in favor of this hypothesis. It would appear, accordingly, that one interpretation of the present results could be that the inverse \% OA vs. \% AA relationship is attributed not only to a direct feedback regulation between the synthesis of this couple of fatty acids, but that ALA, and possibly other fatty acids, may participate to govern the \% OA vs. \% AA relationship. Also our preliminary results obtained in total lipids of sera from 36 male rats suggest that ALA is positively correlated with the $\mathrm{OA} /$ Stear ratio $(\mathrm{r}=0.463, \mathrm{p}=0.004)$, and negatively with the AA/LA ratio $(\mathrm{r}=-0.551, \mathrm{p}<0.001$; results not published). Thus, one explanation of the finding that ALA was positively associated with the OA/AA ratio could be that ALA acts as a stimulator of OA synthesis and /or as an inhibitor of AA synthesis.

On the other hand, there are previous reports which apparently do not seem in support of our findings. Thus, in general, PUFAs like ALA may decrease rather than stimulate stearoyl-CoA desaturase (SCD1) expression and activity [28-32]. From these previous results we might have expected ALA to be inversely - rather than positively - related to the OA/AA and the OA/stearic acid ratios. Apparent discrepancies could possibly be related to differences in approach, for example concerning diet and species used, sex, and whether the study was done in vitro or in vivo. In any instance, the n3 PUFAs including ALA have many functions, and also as yet unknown mechanisms might be involved in their regulation.

We emphasize that the product/precursor ratio used in the present study are only crude estimates of enzyme activities, and more direct methods are needed to clarify whether the suggested mechanisms are valid.

It would appear that many of the alleged positive health effects of OA should be expected if OA acts to counteract effects of, or reduces the relative amounts of AA. Furthermore, health effects of ALA have been attributed to the fact that this fatty acid can be converted to EPA [23]. The associations with ALA studied in the present work do not seem, however, to be explained by a similar mechanism. Since the present analyses are based upon data from a diet trial in chickens, we will not discuss possible health implications of the findings.

The present analyses were carried out on pooled data from a previously published diet trial involving several subgroups [21]. The diets used for the present study contained $2.4 \%$ linseed oil and had equal n6/n3 ratio, but differed in amount of rendered fat, palm oil, red palm oil, and rapeseed oil. Furthermore, in some subgroups the diets had increased amount of selenium [21]. Thus, there was a 2 -fold variation between diets in the content of palmitic acid, and a 3-fold variation in the content of stearic acid. However, in spite of appreciable differences among the diets, the slope of the association curve between ALA and the OA/AA ratio remained unaffected of the diet. For example, a 2-fold increase in the intake of palmitic acid did not influence the OA vs. AA relationship.

We suggest that the consistent inverse relationship between percentages of $\mathrm{OA}$ and AA, possibly governed both by a direct feedback regulation between formation of OA and AA, and also influenced by ALA, could possibly be related to the risk of AA associated conditions and diseases, such as inflammation and cardiovascular diseases. However, the present data are not sufficient to substantiate this hypothesis, and further studies in man are required to examine the possibility. Whatever the mechanisms might be, the present results support our previous observation that percentages of oleic acid and arachidonic acid are inversely related.

\section{Conclusions}

The relative abundances of $\mathrm{OA}$ and $\mathrm{AA}$ in lipids of chicken breast muscle are inversely related. The results are in agreement with previous findings in man and in the rat, raising the question of whether this relationship is a general one across various species. The results suggest that there might be an inverse coordinated regulation of the formation of the two fatty acids, possibly effected by a feedback regulation between the synthesis of OA and AA, and possibly related to ALA (see Figure 3), but further studies are required to clarify whether the hypothesis is valid.

\section{Methods}

The present study is a spin-off of a previously published diet trial [21], in which groups of chickens were fed different types of diet.

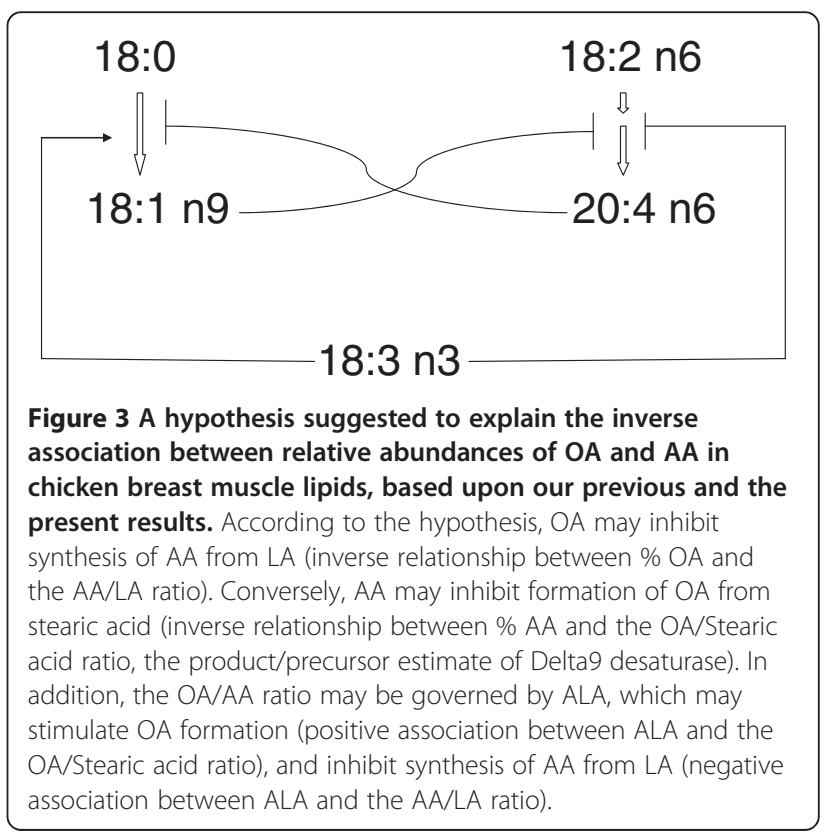


Table 2 Fatty acid composition (\%) of individual diets (see comment below)

\begin{tabular}{|c|c|c|c|c|c|c|}
\hline & C16:0 & C18:0 & C18:1 c9 & C18:2 n6 & C18:3 n3 & n6/n3 \\
\hline $2.4 \% \mathrm{LO}+5.6 \% \mathrm{RF}$ & 18.1 & 10.1 & 28.0 & 19.8 & 14.3 & 1.4 \\
\hline $2.4 \% \mathrm{LO}+5.6 \% \mathrm{PO}$ & 27.9 & 3.3 & 30.2 & 21.7 & 13.7 & 1.6 \\
\hline $2.4 \% \mathrm{LO}+5.6 \% \mathrm{RPO}$ & 28.5 & 3.5 & 29.5 & 21.9 & 13.6 & 1.6 \\
\hline $2.4 \% \mathrm{O}+1.6 \% \mathrm{RO}+4 \% \mathrm{RF}$ & 15.3 & 7.9 & 31.5 & 21.4 & 15.9 & 1.4 \\
\hline $2.4 \% \mathrm{LO}+1.6 \% \mathrm{RO}+4 \% \mathrm{PO}$ & 22.2 & 2.9 & 32.8 & 23.2 & 15.5 & 1.5 \\
\hline
\end{tabular}

$\mathrm{LO}=$ linseed oil; $\mathrm{RF}=$ rendered fat; $\mathrm{PO}=$ palm oil; $\mathrm{RPO}=$ red palm oil; $\mathrm{RO}=$ rapeseed oil.

Note: Since all diets gave the same outcome concerning the present statistical analyses, we only present results of the pooled data in detail.

\section{Ethical approval}

The diet trial in chickens was performed in accordance with National and international guidelines concerning the use of animals in research (Norwegian Animal and Welfare Act, European Convention for the protection of Vertebrate Animals used for Experimental and other Scientific Purposes, CETS No.: 123 1986). The trial was approved by the Regional Norwegian Ethics Committee, and the experimental research followed internationally recognized guidelines.

\section{Chickens and diet}

One day old Ross 308 broiler chickens from Samvirkekylling (Norway) were randomly divided into 10 groups with about 16 birds in each group, and fed equienergetic wheat based diets, from day 1 to 29. The animals had free access to feed and water. They were kept group wise in mesh floored battery cages from day 1 to day 12 , and then placed in separate metabolism cages from day 12 until day 29 . The birds were inspected twice daily by qualified handlers, and every other day by a veterinarian throughout the trial period. The feeds were all containing $8 \%$ supplement fat, of which $2.4 \%$ was linseed oil. The $\mathrm{n} 6 / \mathrm{n} 3$ ratio of the diets was similar, being about 1.5. Content of rendered fat, palm oil, red palm oil and rapeseed oil varied as shown in Table 2. The various diets had identical amounts of the various ingredients, except for the type of fat. In addition, in five subgroups extra selenium was added [21]. Since our preliminary analyses showed that the various diets did not influence the associations studied in the present work, only the results of the pooled sample are presented in detail.

At day 29, the animals were stunned by a sharp blow to the head and killed by exsanguination. Samples from the breast muscle were frozen at $-20^{\circ} \mathrm{C}$ for fatty acid analyses as described by O'Fallon et al. [33].

\section{Determination of fatty acids}

Fatty acid composition of total lipids of breast muscle and feed was determined by gas chromatography [29]. Fatty acid content is presented as $\mathrm{g}$ fatty acid/ $\mathrm{kg}$ tissue (wet weight), and as weight percentage of the measured fatty acids.

\section{Estimates of fatty acid desaturases}

We have used product/precursor ratios as crude estimates of the formation of OA (AA), catalyzed by desaturase/ elongase, i.e. the $\mathrm{OA}$ to stearic acid (Stear) ratio for Delta-9 desaturase (OA synthesis), and the AA/LA ratio to estimate Delta-5/6 desaturase and Elongase-5 (AA formation). Furthermore, we have considered whether the relative abundance of OA (AA) might serve as effectors of a feedback regulation. We accordingly studied 3 bivariate associations: a) the OA/Stear ratio vs. the AA/LA ratio; b) \% OA vs. the AA/LA ratio; and c) \% AA vs. the OA/SA ratio.

\section{Statistical analysis}

On the pooled sample, the relationship between percentages of fatty acids was assessed by correlation (Pearson), and by multiple linear regression. The OA/ AA ratio served as the principal dependent variable and ALA was the independent variable under investigation. We made 4 regression models: Model 1 is without adjustment; Model 2: adjusted for 20:3n3+20:5n3+22:5n3+22:6n3 ; Model 3= Model $2+18: 2$ n6 + 18:3 n6 + 20:2 n6 + 20:3 n6; Model 4 = Model $3+14: 0+14: 1 \quad$ c9 $+15: 0+16: 0+16: 1 \quad c 9+17: 0+$ $18: 0+18: 2 \mathrm{c} 11+18: 2 \mathrm{n} 6+20: 0+20: 1 \mathrm{n} 9+20: 2 \mathrm{n} 6+20: 3$ n6 + unidentified 18:1 isomers (minor amounts) + diet type. Results are presented in tables, or as scatter plots with the regression line included. SPSS 19.0 was used for the regression analyses and Sigma Plot 2001 for producing the figures. A significance level of 0.05 was accepted.

\section{Competing interests}

The authors declare that they have no competing interests.

\section{Authors' contributions}

The present study is a spin-off study of a previously published diet trial, conceived and conducted by AH. ATH conceived and designed the present study, analyzed and interpreted the data, and drafted the article. AH contributed substantially to the interpretation of data and revising the article critically for important intellectual content. Both the authors approved the final version to be published.

\section{Acknowledgements}

The study of which the present one is a spin-off, was funded by grant no 190399 from the Norwegian Research Council, and Animalia; the Norwegian Meat and Poultry Research Centre. We thank the collaborators at the Norwegian University of Life Sciences, and at the Animal Production Experimental Center, Ås, Norway, especially Nicole F. Nyquist, Therese Mosti and Malin Andersen. 


\section{Author details}

${ }^{1}$ Section of Preventive Medicine and Epidemiology, University of Oslo, Norway, Institute of Health and Society, Box 1130 Blindern, 0318 Oslo, Norway. ${ }^{2}$ Department of Animal and Aquacultural Sciences, The Norwegian University of Life Sciences, Box 5003, 1432 Ås, Norway.

Received: 21 February 2014 Accepted: 30 April 2014

Published: 10 May 2014

\section{References}

1. Ryan M, Mclnerney D, Owens D, Collins P, Johnson A, Tomkin GH: Diabetes and the Mediterranean diet: a beneficial effect of oleic acid on insulin sensitivity, adipocyte glucose transport and endothelium-dependent vasoreactivity. QJM 2000, 93:85-91.

2. Gillingham LG, Gustafson JA, Han SY, Jassal DS, Jones PJ: High-oleic rapeseed (canola) and flaxseed oils modulate serum lipids and inflammatory biomarkers in hypercholesterolaemic subjects. $\mathrm{Br} J \mathrm{Nutr}$ 2010, 105:417-427.

3. Damasceno NR, Pérez-Heras A, Serra M, Cofán M, Sala-Vila A, Salas-Salvadó J, Ros E: Crossover study of diets enriched with virgin olive oil, walnuts or almonds: effects on lipids and other cardiovascular risk markers. Nutr Metab Cardiovasc Dis 2011, Suppl. 1:S14-S20.

4. Estévez-González MD, Saavedra-Santana P, López-Ríos L, Chirino R, Cebrero-García E, Peña-Quintana L, Betancor-León P: HDL cholesterol levels in children with mild hypercholesterolemia: effect of consuming skim milk enriched with olive oil and modulation by the TAQ 1B polymorphism in the CETP gene. Ann Nutr Metab 2010, 56:288-293.

5. Cicero AF, Nascetti S, López-Sabater MC, Elosua R, Salonen JT, Nyyssönen K, Poulsen HE, Zunft HJ, Kiesewetter H, de la Torre K, Covas MI, Kaikkonen J, Mursu J, Koenbick C, Bäumler H, Gaddi AV, EUROLIVE Study Group: Changes in LDL fatty acid composition as a response to olive oil treatment are inversely related to lipid oxidative damage: the EUROLIVE study. J Am Coll Nutr 2008, 27:314-320.

6. Cocchi M, Tonello L, Martínez Á, Lercker G, Caramia GM: Extra virgin olive oil and oleic acid. Nutr Clin Diet Hosp 2009, 2009(29):12-24.

7. Terés S, Barceló-Coblijn G, Benet M, Alvarez R, Bressani R, Halver JE, Escribá $P V$ : Oleic acid content is responsible for the reduction in blood pressure induced by olive oil. Proc Natl Acad Sci U S A 2008, 105:13811-13816.

8. Vassiliou EK, Gonzalez A, Garcia C, Tadros JH, Chakraborty G, Toney JH: Oleic acid and peanut oil high in oleic acid reverse the inhibitory effect of insulin production of the inflammatory cytokine TNF-alpha both in vitro and in vivo systems. Lipids Health Dis 2009, 26:25.

9. Reardon M, Gobern S, Martinez K, Shen W, Reid T, McIntosh M: Oleic acid attenuates trans-10, cis-12 conjugated linoleic acid-mediated inflammatory gene expression in human adipocytes. Lipids 2012, 47:1043-1051.

10. Urpi-Sarda M, Casas R, Chiva-Blanch G, Romero-Mamani ES, ValderasMartínez P, Arranz S, Andres-Lacueva C, Llorach R, Medina-Remón A Lamuela-Raventos RM, Estruch R: Virgin olive oil and nuts as key foods of the Mediterranean diet effects on inflammatory biomakers related to atherosclerosis. Pharmacol Res 2012, 65:577-583.

11. Høstmark AT, Haug A: Percentage oleic acid is inversely related to percentage arachidonic acid in total lipids of rat serum. Lipids Health Dis 2013, 12:40.

12. Høstmark AT, Haug A: Percentages of oleic acid and arachidonic acid are inversely related in phospholipids of human sera. Lipids Health Dis 2013, 12:106.

13. Smith $W L$, Murphy RC: The eicosanoids: cyclooxygenase, lipoxygenase and epoxygenase pathways. In Biochemistry of Lipids. Edited by Vance DE, Vance JE. U.K: Elsevier; 2008:331-362

14. Moncada S, Higgs EA: Arachidonate metabolism in blood cells and the vessel wall. Clin Haematol 1986, 15:273-292

15. Mayes PA: Metabolism of unsaturated fatty acids and eicosanoids. In Harper's Biochemistry. 25th edition. Edited by Murray RK, Granner DK, Mayes PA, Rodwell WW. New York: McGraw-Hill; 2000:250-258.

16. Alvheim AR, Malde MK, Osei-Hyiaman D, Lin YH, Pawlosky RJ, Madsen L, Kristiansen K, Frøyland L, Hibbeln JR: Dietary linoleic acid elevates endogenous 2-AG and anandamide and induces obesity. Obesity (Silver Spring) 2012, 20:1984-1994.

17. Lowry RR, Tinsley IJ: Oleic and linoleic acid interaction in polyunsaturated fatty acid metabolism in the rat. J Nutr 1966, 88:26-32.
18. Haug A, Olesen I, Christophersen OA: Individual variation and intraclass correlation in arachidonic acid and eicosapentaenoic acid in chicken muscle. Lipids Health Dis 2010, 15:37.

19. Miyazaki M, Ntambi JM: Fatty acid desaturation and chain elongation in mammals. In Biochemistry of Lipids. Edited by Vance DE, Vance JE. U.K: Elsevier; 2008:191-211.

20. Ntambi JM, Bené H: Polyunsaturated fatty acid regulation of gene expression. J Mol Neurosci 2001, 16:273-278.

21. Haug A, Nyquist NF, Mosti TJ, Andersen M, Høstmark AT: Increased EPA levels in serum phospholipids of humans after four weeks daily ingestion of one portion chicken fed linseed and rapeseed oil. Lipids Health Dis 2012, 11:104

22. Hu FB, Stampfer MJ, Manson JAE, Rimm EB, Wolk A, Colditz GA, Hennekens $\mathrm{CH}$, Willett WC: Dietary intake of a-linolenic acid and risk of fatal ischemic heart disease among women. Am J Clin Nutr 1999, 69:890-897.

23. Connor WE: Alpha linolenic acid in health and disease. Am J Clin Nutr 1999, 69:827-828.

24. Rodriguez-Leyva D, Bassett CMC, McCullough R, Pierce GN: The cardiovascular effects of flaxseed and its omega-3 fatty acid, alpha-linolenic acid. Can J Cardiol 2010, 2:489-496.

25. Kudo N, Yamazaki T, Sakamoto T, Sunaga K, Tsuda T, Mitsumoto A, Kawashima $Y$ : Effects of perfluorinated fatty acids with different carbon chain length on fatty acid profiles of hepatic lipids in mice. Biol Pharm Bull 2011, 34:856-864.

26. Ringbom T, Huss U, Stenholm A, Flock S, Skattebøl L, Perera P, Bohlin L: Cox-2 inhibitory effects of naturally occurring and modified fatty acids. J Nat Prod 2001, 64:745-749.

27. Schisterman EF, Cole SR, Platt RW: Overadjustment bias and unnecessary adjustment in epidemiologic studies. Epidemiology 2009, 20:488-495.

28. Telle-Hansen VH, Larsen LN, Høstmark AT, Molin M, Dahl L, Almendingen K Ulven SM: Daily intake of cod or salmon for 2 weeks decreases the $18: 1 \mathrm{n}-9 / 18: 0$ ratio and serum triacylglycerols in healthy subjects. Lipids 2012, 47:151-160.

29. Novak EM, Innis SM: Impact of maternal dietary $n-3$ and n-6 fatty acids on milk medium-chain fatty acids and the implications for neonatal liver metabolism. Am J Physiol Endocrinol Metab 2011, 301:E807-E817.

30. McNamara RK, Magrisso IJ, Hofacer R, Jandacek R, Rider T, Tso P, Benoit SC: Omega-3 fatty acid deficiency augments risperidone-induced hepatic steatosis in rats: positive association with stearoyl-CoA desaturase. Pharmacol Res 2012, 66:283-291.

31. Manickam E, Sinclair AJ, Cameron-Smith D: Suppressive actions of eicosapentaenoic acid on lipid droplet formation in 3 T3-L1 adipocytes. Lipids Health Dis 2010, 9:57.

32. Zhang J, Vanden Heuvel J, Kris-Etherton P: Alpha linolenic acid increases lipid efflux through inhibition of stearoyl CoA desaturase 1 during foam cell formation. FASEB J 2008, 22:691.7. Meeting Abstract

33. O'Fallon JV, Busboom JR, Nelson ML, Gaskins CT: A direct method for fatty acid methyl ester synthesis: application to wet meat tissues, oils, and feedstuffs. J Anim Sci 2007, 85:1511-1521.

\section{doi:10.1186/1476-511X-13-76}

Cite this article as: Høstmark and Haug: The inverse association between relative abundances of oleic acid and arachidonic acid is related to alpha -linolenic acid. Lipids in Health and Disease 2014 13:76.

\section{Submit your next manuscript to BioMed Central and take full advantage of:}

- Convenient online submission

- Thorough peer review

- No space constraints or color figure charges

- Immediate publication on acceptance

- Inclusion in PubMed, CAS, Scopus and Google Scholar

- Research which is freely available for redistribution 